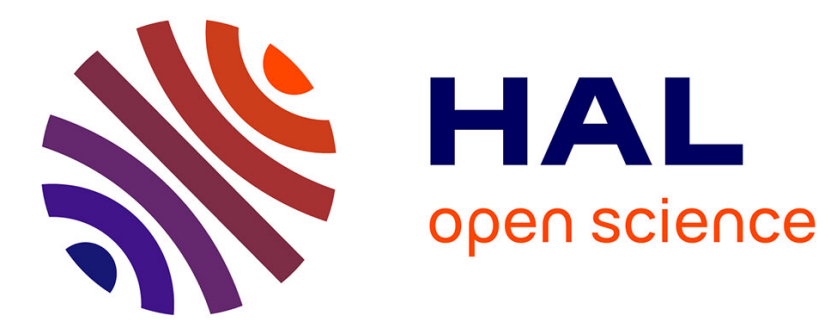

\title{
Sur la mesure du potentiel périodique interne des cristaux
}

\author{
A. Hautot, J.J. Trillat
}

\section{To cite this version:}

A. Hautot, J.J. Trillat. Sur la mesure du potentiel périodique interne des cristaux. J. Phys. Radium, 1938, 9 (4), pp.133-139. 10.1051/jphysrad:0193800904013300 . jpa-00233569

\section{HAL Id: jpa-00233569 https://hal.science/jpa-00233569}

Submitted on 1 Jan 1938

HAL is a multi-disciplinary open access archive for the deposit and dissemination of scientific research documents, whether they are published or not. The documents may come from teaching and research institutions in France or abroad, or from public or private research centers.
L'archive ouverte pluridisciplinaire HAL, est destinée au dépôt et à la diffusion de documents scientifiques de niveau recherche, publiés ou non, émanant des établissements d'enseignement et de recherche français ou étrangers, des laboratoires publics ou privés. 


\title{
SUR LA MESUURE DU POTENTIEL PÉRIODIQUE INTERNE DES CRISTAUXX
}

\author{
Par A. HAUTOT.
}

Chargé de cours à l'Université de Liége,

et J. J. TRILLAT.

Professeur à la Faculté des Sciences de Besançou.

\begin{abstract}
Sommaire. - Cet article contient l'exposé du principe d'une méthode de mesure des termes $V_{0 . ;}$ lu développement en série de Fourier du potentiel périodique interne descristaux (cubiques) ainsi qu'un tssai de la réalisation expérimentale de celte mélhode Des mesures ont été faites pour $\mathrm{Ni}, \mathrm{Au}, \mathrm{Cu}{ }_{2} \mathrm{O}$ el le graphite. Les valeurs obtenues ne s'accordent pas du tout avec la théorie acluelle de la réflexion d'une onde électronique par un cristal; les auteurs indiquent dans quel sens il convient de modifier celle théorie pour obtenir des résultats en accorl avec les mesures
\end{abstract}

1. Potentiel moyen interne du cristal. - Soit un faisceau d'électrons monocinétiques d’énergie $E$, exprimée en volts. On sait que - abstraction faite de la correction de relativité - la loi de Bragg classique

$$
2 d \sin \theta=k \lambda=k \frac{\sqrt{150}}{\sqrt{E}}
$$

relative aux réflexions sélectives de ce faisceau par un cristal, n'est valable qu'en première approximation. L'existence d'un potentiel moyen $V_{0}$ à l'intérieur du cristal conduit à lui substituer l'expression

$$
2 d \sin \theta^{\prime}=k \lambda^{\prime}=k \frac{\lambda}{\mu} .
$$

Tout se passe donc comme si l'onde électronique subissait à l'entrée du cristal une réfraction avec l'indice $\mu$ :

$$
\mu=\frac{\cos \theta}{\cos \theta^{\prime}}=\sqrt{1+\frac{V_{0}}{E}} .
$$

Pour des électrons de grande énergie cinétique, le terme $V_{0}$ étant tout à fait négligeable devant $\boldsymbol{E}$, la loi de Bragg ordinaire (1) est parfaitement applicable; mais la valeur de l'indice de réiraction augmentant à mesure que l'énergie cinétique des électrons diminue, les écarts à la loi de Bragg deviennent appréciables pour des électrons lenıs. Aussi l'étude de la diffıaction des électrons lents par les crislaux a t-elle permis de mesurer l'indice de réfraction et, partant, le potentiel moyen interne $V_{0}$ des cristaux. Des formules ci-dessus on tire en effet aisément la valeur de $V_{0}$ :

$$
V_{0}=-E \sin ^{2} \theta+150 \frac{l^{2}}{4 d^{2}} \text {. }
$$

En fait, les mesures de $V_{0}$ sont affectées d'erreurs assez importantes dont témoignent d'ailleurs les valeurs différentes données par les auteurs. Nous rappellerons seulement ici les mesures concernant deux métaux dont nous nous occuperons par la suite.

Pour le nickel, Davisson et Germer (1) obtiennent la valeur $V_{0}=-15 \mathrm{~V}$, moyenne d'une quinzaine de mesures donnant des valeurs comprises entre - 10 et $-28 \mathrm{~V} ;$ Rupp $\left(^{2}\right)$ donne $V_{0}=-16 \mathrm{~V}$, moyenne de 10 mesures comprises entre $-14 \mathrm{et}-20 \mathrm{~V}$.

Pour l'or, Rupp (') donne : $V_{0}=-14 \mathrm{~V}$.

2. Potentiel périodique interne. - En fait, au sein d'un cristal, le potentiel est une fonclion triplement périodique dont les périodes sont les dimensions de la maille élémentaire. Nous nous limiterons au cas du réseau cubique d'arète $d$; alors $V$ s'écrit sous la forme d'une série de Fourier

$$
V=\sum_{\alpha, s ;} V_{\alpha, z ;} \mathrm{e}^{+\frac{2 \pi \mathrm{i}}{d}(\alpha x+\xi y+; \bar{s})},
$$

$\alpha, \beta, \gamma$ étant des nombres entiers; ce sont les indices des différents plans réticulaires du cristal.

Les coeflicients $V_{\alpha, \beta ;}$ s'obtiennent par la formule

$$
V_{o s i}=\frac{1}{L} \int_{L} V(x, y, z) \mathrm{e}^{-\frac{2 \pi \mathrm{i}}{d}(\alpha x+\beta y+; z)} \mathrm{d} L
$$

$L$ étant le volume du cristal.

Le potentiel moyen dont il est question au $\$ 1$ est le premier terme de ce développement :

$$
V_{0} \equiv V_{000}=\frac{1}{L} \int_{L}^{v} V(x, y, z) \mathrm{d} L
$$

Le but du présent article est de donner le principe d'une méthode de mesure des coefficients $V_{\%:}$ autres que $V_{000}$, relatifs aux fluctuations du potentiel dans le cristal ; ces termes n’ont en effet pas encore été mesurés jusqu'à présent. Auparavant, rappelons succinctement une méthode théorique permettant de calculer ces termes.

3. Calcul des termes successifs du développement en série de Fourier du potentiel interne d'un cristal. - Cette méthode ramène le calcul des termes $V_{a,: ;}$ à celui de la densité électrique $\rho$ par emploi de l'équation de Poisson

$$
\Delta V=-4 \pi p \text {. }
$$


En effet, l'expression

$$
V_{\alpha \beta \eta \gamma}=\frac{1}{L} \int_{L} V \mathrm{e}^{-\frac{2 \pi \mathrm{i}}{d}(\alpha \cdot x+\beta y+\gamma \bar{s}} \mathrm{d} L
$$

peut s'écrire en intégrant partiellement 2 fois par rapport à $x$ et en tenant compte de ce que $V$ et $\frac{\partial V}{\partial x}$ sont nuls à la surface du cristal :

$$
V_{\alpha \beta \gamma}=-\frac{1}{L} \frac{d^{2}}{4 \pi^{2} \alpha^{2}} \int_{L} \frac{\partial^{2} V}{\partial x^{2}} \mathrm{e}^{-\frac{2 \pi \mathrm{i}}{d}\left(\alpha x+q y+\gamma^{z}\right)} \mathrm{d} L .
$$

On obtient deux expressions analogues en intégrant par rapport à $y$ et $z$; en combinant le résultat des trois intégrations, on trouve immédiatement

$$
\begin{aligned}
V_{u, i} & =-\frac{1}{L} \frac{d^{2}}{4 \pi^{2}\left(\alpha^{2}+\beta^{2}+\gamma^{2}\right)} \int_{L} \Delta V \mathrm{e}^{-\frac{2 \pi \mathrm{i}}{d}(u x+\xi y+\gamma ;)} \mathrm{d} L \\
& =\frac{1}{L} \frac{a^{2}}{\pi\left(\alpha^{2}+\beta^{2}+\gamma^{2}\right)} \int_{L} \rho \mathrm{e}^{-\frac{2 \pi \mathrm{i}}{d}(\alpha x+\beta y+; \bar{y})} \mathrm{d} L
\end{aligned}
$$

A cause dela périodicité de la fonction $p$ dans le cristal, on peut écrire :

$$
V_{\sigma \xi \gamma}=\frac{1}{l_{0}} \frac{d^{2}}{\pi\left(\alpha^{2}+\beta^{2}+\gamma^{2}\right)} \int_{l_{0}} \rho \mathrm{e}^{-\frac{2 \pi \mathrm{i}}{d}(u x+y y+\gamma z)} \mathrm{d} L,
$$

$l_{0}$ étant le volume de la maille élémentaire du cristal.

Dans un cube à faces centrées, la maille élémentaire contient 4 atomes dont les coordonnées sont $r_{j}\left(x_{j}, y_{j}\right.$, $\left.z_{j}\right)$ avec $j=1,2,3$, 4 .

On démontre sans peine $\left(^{*}\right)$ l'égalité

$$
\rho=\frac{1}{l_{0}} \sum_{j} \mathrm{e}^{\frac{2 \pi \mathrm{i}}{a}\left(\sigma, r_{j}\right)} \int_{l_{0}} \rho_{1}(r) \mathrm{e}^{-\frac{2 \pi \mathrm{i}}{d}(\sigma, r)} \mathrm{d} \boldsymbol{L},
$$

$p_{1}(r)$ étant la contribution d'un seul atome à la densité électrique. Celle-ci est la somme de deux termes, l'un $\rho_{z}$ dù au noyau etl'autre $f_{c}$ dû aux couches électroniques. En fixant l'origine des coordonnées au noyau, l'exponentielle vaut 1 pour le noyau; plus $p_{z}$.d $L=$ - $Z e$, en appelant $Z$ le nombre atomique de l'élément. La contribution du noyau au terme $V_{\alpha \beta \gamma}$ est donc égale à

$$
-\frac{1}{l_{0}} \sum_{j} \mathrm{e}^{\frac{2 \pi \mathrm{i}}{d}\left(u, r_{j}\right)} \frac{d^{2} Z e}{\pi\left(\alpha^{2}+\beta^{2}+\gamma^{2}\right)} .
$$

Le calcul de la densité électronique $p_{e}$ a été fait par deux méthodes différentes : la méthode de ThomasFermi $\left({ }^{3}\right)$ et la méthode de Bethe $\left({ }^{4}\right)$. En appelant $e F$ la contribution des couches électroniques à la valeur de l'intégrale, on obtient:

$$
V_{u, \beta \gamma}=\frac{1}{l_{0}} \sum_{j} \mathrm{e}^{\frac{2 \pi \mathrm{i}}{d}\left(\alpha, r_{j}\right)} \frac{d^{2} e}{\pi\left(\alpha^{2}+\beta^{2}+\gamma^{2}\right)}(Z-F) .
$$

En calculant $\rho_{e}$ par la méthode de Thomas-Fermi, on

(`) Cf. ex. Frö̈Licн. Elektronentheorie der Metalle, p. 370. trouve pour le nickel :

$$
r_{111}=-16,5 \mathrm{~V} ; \quad V_{200}=V_{020}=V_{002}=-12 \mathrm{~V} ;
$$

la méthode de Bethe donne :

$$
V_{111}=-13,4 ; \quad V_{200}=-12,6 \mathrm{~V} .
$$

Pour l'or, la méthode de Thomas-Fermi nous a donné :

$$
V_{111}=-18 \mathrm{~V} \text {. }
$$

4. Principe de la méthode de mesure. - La méthode repose sur l'emploi d'une formule relative à la réflexion d'une onde électronique par un cristal; cette onde électronique obéit dans le cristal à l'équation de Schrödinger où $V$ est le potentiel périodique donné par (ð) ; dans ce cas, Peierls et Brillouin ont obtenu la solution de l'équation de Schrödinger par approximations successives. La réflexion de cette onde électronique par le cristal a été étudiée théoriquement par Bethe et Kikuchi $\left(^{3}\right)$; ceux-ci expriment les conditions de continuité auxquelles doit satisfaire l'onde à la surface du cristal, savoir :

$$
\left.\begin{array}{r}
\varphi_{i=0}=\psi_{z=0} \\
\left(\frac{\partial \varphi}{\partial z}\right)_{i=0}=\left(\frac{\partial \psi}{\partial z}\right)_{i=0}
\end{array}\right\}
$$

$\psi$ et $\psi$ étant les fonctions d'ondes à l'exlérieur et à l'intérieur du cristal. Ces conditions permettent de calculer le pouvoir réflecteur du cristal; Kikuchi obtient les résultats suivants :

$1^{\circ}$ En dehors des zones de réflexion sélectives de Bragg, le pouvoir réflecteur est donné par la formule

$$
R=1-\frac{W}{\sqrt{W^{2}+1}},
$$

$W$ étant une grandeur dépendant de l'énergie de l'onde électronique, de l'angle d'incidence el des constantes cristallines. En général, $W$ est très supérieur à 1, de sorte qu'on a alors :

$$
R \sim 0 .
$$

$2^{\circ}$ Pour une zone de réflexion sélective, on a sensiblement : $R=1$; il y a réflexion totale; cette zone de réflexion totale a d'ailleurs une certaine largeur angulaire $\Delta \theta$ donnée par la formule

$$
2 \Delta \theta \cdot \theta \cdot \boldsymbol{E}=V_{u, \beta \gamma}
$$

où $E$ ' est l'énergie de l'onde électronique, o l'angle de Bragg pour le plan $\alpha, \beta, \gamma$ et $V_{\alpha, \beta}$ le terme correspondant du développement en série de Fourier.

On peut traduire ces résultats par le graphique ci-contre obtenu en traçant la variation du pouvoir réflecteur $R$ é fonction de l'angle d'incidence $\theta$; la courbeci-contre se rapporte à la réflexion d'une onde électronique par les plans $111 \mathrm{de} \mathrm{Ni}$

Nous avons fait les mesures de $\Delta \theta$ pour différents anneaux de diffraction donnés par plusieurs cristaux; l'application de la formule (13) de Kikuchi conduit $\dot{a}$ 
des valeurs des termes $V_{a \beta \gamma}$ de l'ordre d'une fraction de volt, c'est-à-dire à une valeur cent fois plus petite que celle qui résulte du calcul théorique du\$3. Nous avons recherché les causes de cet écart considérable et avons été amenés à abandonner la formule (13) et à lui substituer la formule toute différente

$$
V_{u, y}=\frac{\Delta \theta}{0} \cdot E\left(^{*}\right)
$$

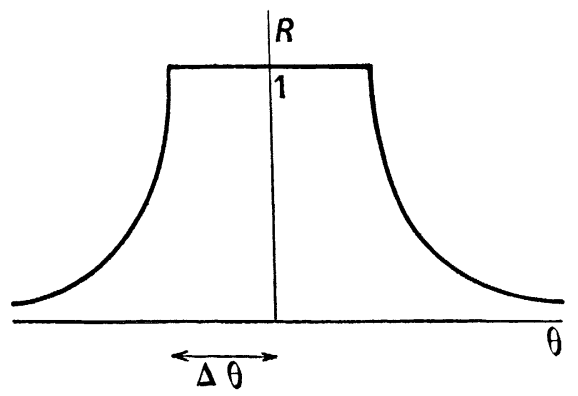

Fig. 1.
5. - Démontrons la formule (14) à partir d'un résultat classique de la théorie des métaux.

Peierls et Brillouin ont démontré que lorsqu'une onde électronique se propage dans un cristal, 2 cas se présentent :

$1^{\circ}$ L'énergie de l'onde est telle qu'aucune réflexion sélective ne puisse se produire; l'onde électronique se propage alors avec une bonne approximation comme une onde électronique libre.

$2^{\circ} \mathrm{Au}$ voisinage d'une réflexion de Bragg sur un plan $\alpha, \beta, \gamma$, l'onde électronique ne peut plus être considérée comme libre; notamment, il existe pour l'énergie une discontinuité de largeur $\Delta E=2 V_{\alpha \beta \gamma}$; en d'autres termes, l'onde électronique d'énergie comprise entre $E$ et $E+\Delta E$ ne peut se propager dans le cristal; il y a donc réflexion totale de cette onde sur le plan $\alpha \beta \gamma$.

Ce dernier résultat va nous permettre d'établir immédiatement la formule (14).

L'équation de Bragg

donne par différentiation

$$
2 d \sin \theta=k \frac{h}{\sqrt{2 m E}}
$$

$$
\frac{|\Delta \theta|}{\operatorname{tg} \theta}=\frac{1}{2} \frac{|\Delta E|}{E}
$$

(*) Dans une note publiée aux $C . R . A c . S c .$, Paris, 1937, t. 205, p. 1161, A. Havtor avait donné les valeurs des coefficicnts $V_{\alpha^{\circ}}$., calculés à purtir de la formule (13) de Bethe et Kikuchi; A. Hautó signalait d'autre part la divergence énorme entre les valeurs ainsi obtenues et celles que donnent les méthodes théoriques de Bethe ou de Thomas-Fermi. La formule (13) de Bethe et Kikuchi paraissant ne pas traduire correctement le phénomène de réflexion sélective d'une onde électronique par un cristal, il y a donc lieu d'abandonner les valeurs des termes $V_{a, 3 \%}$ auxquelles A. Hautot avait été conduit par emploi de cette for mule; comme nous le montrerons plus loin, la formule (14) est susceptible de conduire à des résultats bien plus satisfaisants.
En combinant cette relation avec la relation

$$
\Delta E=2 V_{a:-}
$$

valable pour une réflexion sélective, on a immédiatement :

$$
\left|V_{\alpha \beta Y}\right|=E \frac{|\Delta \theta|}{\operatorname{tg} \theta}
$$

Dans le cas d'électrons rapides, $\theta \# \operatorname{tg} \theta$ et on a la formule (14).

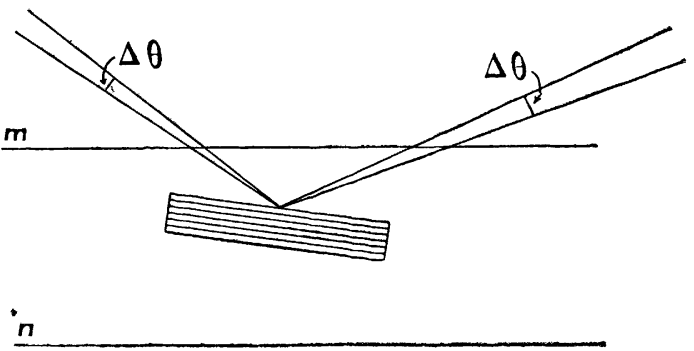

Fig. 2.

Choisissons un cristal élémentaire appartenant à une feuille polycristalline $\mathrm{mn}$; l'angle $\Delta \theta$ figurant dans cette formule est le domaine de réflexion correspondant aux plans $\alpha, \beta, \gamma$ du cristal; l'angle $\Delta^{\prime} \theta$ observé à l'extérieur du cristal sera identique à $\Delta \theta$ si l'on néglige la réfraction à la sortie de la feuille cristalline; or, celle-ci est tout à fait négligeable car on sait que, pour des électrons rapides, l'indice de réfraction est pratiquement égal à $\left.1{ }^{*}{ }^{*}\right)$. C'est donc à partir de la formule (14), et non de la formule (13) de Kikuchi, que nous calculerons la valeur des termes $V_{\alpha, \beta}$; la méthode de mesure de ces termes consiste donc à mesurer la valeur de $\Delta \theta$ correspondant à chaque terme $V_{\alpha \beta \gamma}$ et $\grave{a}$ introduire la valeur obtenue dans (14).

\section{Réalisations expérimentales.}

6. Conditions générales. - On a utilisé la méthode de diffraction électronique par transmission à travers une feuille mince, d'une épaisseur de quelques centaines d'Angström. Une telle feuille contient en effet un nombre considérable de microcristaux, ce qui permet, pour des électrons monocinétiques, d'enregistrer tous les anneaux correspondant aux différents plans réticulaires; on peut obtenir ainsi sur un même

(*) Comme on le voit, nous négligeons ici les phénomènes susceptibles de se produire à la surface da cristal; comme le montre J. Farineau (voir ce même numéro du Journal de Physique, p. 157), ce sont les conditions de con tinuité que l'on impose à l'onde à la surface du cristal qui donnent la formule (13) en désaccord avec nos expériences. Remarquons d'autre part que si la théorie de Bethe-Kikuchi conduit à une va'eur beaucoup trop grande de la largeur $\Delta \theta$ des anneaux de diffraction électronique et des termes $V_{a \cdots}$, elle donne par contre la valeur correcte (4) du potentiel moyen $V_{0}$ et de lindice de réfraction $\mu$; ceci provient de ce que les conditions de continuité imposées à l'onde électronique à son entrée dans le cristal n'interviennent en fait pas dans le calcul de $V_{0}$ et de $\mu$. 
cliché les mesures des différents termes $V_{\alpha: \cdots}$ correspondant à tous les anneaux observés.

Pour obtenir une mesure correcte de la largeur $\Delta \theta$ des anneaux de diffraction (qui est souvent très petite, de l'ordre de $\mathbf{~}^{\prime}$ ), il faudrait pouvoir opérer avec un faisceau électronique de section ponctuelle; sinon, à la largeur des anneaux due aux fluctuations $V_{\alpha, \cdots}$ du potentiel interne du cristal se superpose la largeur même du faisceau électronique. Nous avons donc cherché à réaliser un faisceau incident aussi fin que possible et rigoureusement parallèle; car si le faisceau incident comporte une divergence $\Delta \theta$ même très faible, elle contribue aussi à un élargissement parasite des anneaux de diffraction. Dans le $\$ 6$, nous montrons comment ont été réalisées ces deux conditions essentielles.

Il importe aussi que la tension $E$ accélératrice des électrons soit rigoureusementconstante, car une variation $\Delta \boldsymbol{E}$ de celle-ci donne un élargissement des anneaux. Nous discutons ce point dans le $\$ 7$.

Nous montrons ensuite comment nous avons éliminé une autre cause d'élargissement dù à des champs magnétiques parasites.

Le $\$ 9$ est consacré à l'étude des causes d'élargissement qu'il est difficile et mème impossible de faire disparaître complètement; nous montrons comment nous procédons pour mesurer cet élargissement résiduel (d'ailleurs très faible) et comment nous pouvons ainsi corriger les résultats bruts des mesures.

Nous exposons ensuite la technique des mesures et discutons la précision des résultats.

7. Réalisation d'un faisceau électronique paralIèle et très fin. - Le collimateùr employé pour canaliser les électrons a $12 \mathrm{~cm}$ de longueur et porte à ses extrémités deux diaphragmes ayant une largeur de $0,03 \mathrm{~mm}$; malgré la très faible section de ce collimateur, les phénomènes de diffraction électronique restent bien visibles sur l'écran fluorescent. Le faisceau incident ainsi obtenu est très fin et présente une divergence extrêmement faible; sa largeur et sa divergence ont été mesurées par les denx procédés suivants.

1 Le faisceau électronique est reçu directement sur la plaque photographique. On donne à celle-ci un mouvement de rotation rapide et on obtient ainsi sur la plaque un trait circulaire dont la largeur est égale au diamètre du faisceau. Le but de ce mouvement rapide est d'éviter la surexposition de la plaque car il en résulterait inévitablement un élargissement notable du trait dû aux phénomènes de diffusion dans la couche sensible. Un très grand nombre de mesures ont donné un diamètre constant de $0,11-0,12 \mathrm{~mm}$, soit une section de $0,01 \mathrm{~mm}^{2}$ environ : c'est donc la section du faisceau frappant la plaque photographique.

$2^{\circ}$ Le faisceau électronique traverse un film mince amorphe $A$ (fig. 3 ) qui diffuse les électrons dans toutes les directions; puis un second film mince $B$; celui-ci, constitué par une feuille d'or battue puis amincie, porte des trous microscopiques $o$; chacun de ces trous agit vis-à-vis du faisceau transmis par $A$ comme un objectif- point. Il se forme ainsi sur la plaque photographique $\boldsymbol{P}$ une série d'images de la section $m n$ du faiscèau, agrandies dans le rapport $\frac{L}{l}$. On a donc:

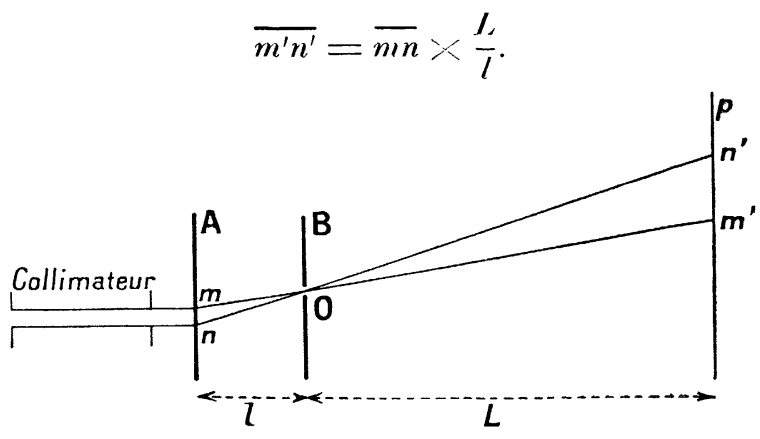

Fig. 3.

Cette deuxième méthode, très précise, donne pour le diamètre du faisceau en $A$ une largeur plus faible, égale à 0,113 à $0,04 \mathrm{~mm}$; d'où l'on peut conclure que le faisceau électronique passant de $A$ en $P$ subit un léger élargissement (de l'ordre de $0,07 \mathrm{~mm}$ ) dû à des causes que nous analyserons plus loin $\left({ }^{*}\right)$.

Le faisceau électronique présente donc entre $A$ et $P$ une divergence $\Delta_{1} \theta$ très faible que l'on peut calculer par la formule

On a :

$$
2 \Delta_{1} \theta=\frac{\Delta r}{L}=\frac{0,07}{300}
$$

$$
\Delta_{1} \theta \sim \frac{1^{\prime}}{3}
$$

Malgré la finesse et le parallélisme excellent de ce faisceau, il n'est cependant pas possible de le considérer comme rigoureusement ponctuel et parallèle; nous indiquerons ci-dessous comment nous avons procédé pour corriger les mesures de cette cause d'erreur d'ailleurs assez petite.

8. Etude de la variation de tension. - Une variation $\Delta E$ de la tension accélératrice $E$ des électrons

(*) Ces deux méthodes sont très précises; la seconde pourrait rendre des services dans les nombreux problèmes où l'on utilise des faisceaux électroniques (microscope électronique et oscillographe); elle permet en effet non seulement de mesurer avec précision la largeur eu faisceau mais donne encore la forme de la section du faisceau; par exemple, si le collimateur employé pour canaliser les électrons porte des trous non circulaires, le faisceau électronique a aussi une section non circulaire; l'examen des images données par ce faisceau sur la plaque photographıque perm+t ainsi de parlaire l'état du collimateur. Enfin, il paraît intéressant de signaler que cette méthode permet encore de connaitre la répartition des électrons dans une section du faisceau: les électrons constituant le faisceau exercent en efret l'un sur l'autre des actions répulsives; il en résulte que la densité électronique doıt être minimum suivant l'axe du faiscaau et maximum au voisinage des bords; la methode ci-dessus permet de vérifier très bien celte prévision: sur la plaque photographique, le centre du spot apparait en effet en blanc (endroit non impressionné) alors que les bords du spot ont le noircissement maximum. 
donne un élargissement parasite des anneaux de diffraction que l'on peut calculer en dérivant la formule

()n lrouve :

$$
l \frac{h}{\sqrt{2 m e V}}=2 d \sin 0 \text {. }
$$

$$
|\Delta \theta|=\frac{1}{2}\left|\frac{\Delta V}{V}\right| \operatorname{tg} \theta \text {. }
$$

Soit $r$ le rayon de l'anmeau de diffraction, $D$ la distance de la lame cristalline à la plaque; dans nos expériences, $D=30,7 \mathrm{~cm}$; on a :

$$
2 \theta=\frac{D}{r} ; \Delta r=2 D . \Delta \theta
$$

et par conséquent :

$$
|\Delta r|=D\left|\frac{\Delta V}{V}\right| \operatorname{tg} \theta
$$

$\Delta V$ est le résidu alternatif de la tension redressée appliquée au tube électronique; nous le mesurons en plaçant le tube électronique dans les conditions de fonctionnement utilisées pour la photographie des anneaux de diffraction ( $V=40 \mathrm{kV}, i=3-4$ millis). Nous plaçons en dérivation sur le tube électronique une capacité connue et mesurons le débit dans ce condensateur; nous obtenons ainsi l'intensité alternative traversant le condensateur; cette intensité étant très nettement inférieure à celle qui traverse le condensateur quand on lui applique une différence de potentiel alternative de $100 \mathrm{~V}$, nous pouvons conclure que $\Delta V$ reste très inférieure à $100 \mathrm{~V}$.

D'où :

$$
\left|\frac{\Delta V}{V}\right|<<\frac{100}{40.000}<<\frac{2, \mathfrak{5}}{1000} .
$$

Il importe également que la tension primaire du transformateur $(110 \mathrm{~V})$ reste constante au cours de la prise des clichés. A cet effet, un voltmètre est placé sur ce circuit et un rhéostat permet de régler la tension primaire de manière qu'au cours de la prise des clichés, la variation ne dépasse pas $0,1 \mathrm{~V}$; il en résulte une variation $\frac{\Delta V}{V}$ ne dépassant pas 1/1 000 .

Au total, $\Delta V$ ne dépasse certainement pas $2 / 1000$ à $3 / 1000$; il s'agit là d'ailleurs d'une valeur maximum de l'erreur, probablement non atteinte au cours des mesures; on peut estimer à quelques centièmes de $\mathrm{mm}$ l'élargissement des anneaux résuitant de cette variation de lension.

Un voltmètre électrostatique branché sur le circuit secondaire du transformateur permet d'ailleurs, à chaque instant, de contrôler la constance de la tension.

9. Elimination des champs magnétiques parasites. - Ces champs magnétiques sont dus à deux causes.

$1^{\circ}$ Le champ magnétique terrestre. Celui-ci produit une déformation constante (d'ailleurs extrêmement petite pour des électrons rapides) de la trajectoire décrite par le faisceau électronique, mais elle n'en modifie pas la largeur; nous ne nous en préoccupons donc pas.

$2^{\circ}$ Les champs magnétiques dus à l'appareillage électrique utilisé par la production de vide et de la haute tension. Ces champs magnétiques sont variables puisqu'ils sont dus à des courants alternatifs; ils produisent donc une déformation périodique rapide de la trajectoire du faisceau électronique; le spot obtenu sur l'écran fluorescent ou la plaque photographique est alors le résultat des positions successives du faisceau; il est donc élargi. - L'extrême finesse du faisceau employé permettait effectivement d'observer cet élargissement, égal à quelques centièmes de $\mathrm{mm}$.

Nous avons fait disparaître complètement ces champs magnétiques parasites en écartant à de grandes distances de l'analyseur électronique tout l'appareillage électrique parcouru par des courants alternatifs; l'opérateur dirigeait alors le fonctionnement de l'analyseur électronique au moyen de commandes télémécaniques.

10. Causes d'élargissement résiduel du faisceau électronique. $-1^{\circ}$ Il faut d'abord rappeler que le faisceau incident a une largeur qui, bien que très faible, n'est pas négligeable : elle est de $0,03 \mathrm{~mm}$ environ à l'endroit où se place la feuille diffractant les électrons.

$2^{\circ}$ Le faisceau présente en outre, entre cette feuille et la plaque photographique, une légère divergence, de l'ordre de $1 / 3$ '. Celle ci est due vraisemblablement aux deux phénomènes suivants qui sont inévitables :

a) Le gaz résiduel produit une certaine diffusion des électrons.

b) Les électrons constituant le faisceau électronique exercent l'un sur l'autre des actions répulsives. Ce problème a été traité théoriquement par E. E. Watson $\left(^{6}\right)$; sans insister sur ce point, signalons que cet effet peut-être minimisé en opérant, comme dans nos essais, avec un débit faible $(0,1$ milli dans l'analyseur électronique proprement dit) et sous une tension élevée $(40 \mathrm{kV})$.

$3^{\circ}$ Il faut encore considérer l'élargissement dû à la diffusion des électrons dans l'émulsion.

En vue de connaître l'élargissement dù à ces causes, on a photographié sur chaque cliché le spot constituant la section du faisceau électronique par la plaque. A cet effet, on imprime à la plaque un mouvement rapide de rotation; le spot dessine sur la plaque un trait circulaire dont la largeur mesure le diamètre du faisceau, compte tenu des phénomènes de diffusion dans la couche sensible : la vitesse de rotation de la plaque est telle en effet que le noircissement du spot direct soit sensiblement le mème que celui des anneaux.

On a ainsi sur chaque cliché la possibilité de mesurer la largeur de chaque anneau et la largeurdu spot direct; la différence donne la largeur vraie de l'anneau.

11. Mesures des largeurs des anneaux. - Ces mesures ont été faites par deux procédés : 
1. Mesure directe au comparateur;

2 Mesure faite au moyen d'un microphotomètre enregistreur. Dans ce cas, on a réduit au minimum l'inertie de l'appareil; celle-ci n'étant cependant pas négligeable, on a apporté aux mesures brutes une correction obtenue en microphotométrant une fente étalonnée de $0,2 \mathrm{~mm}$; soit une largeur voisine de celle des anneaux.
Ces deux méthodes ont d'ailleurs donné des résultats concordants; les nombres indiqués dans les tableaux ci-dessous sont les moyennes de nombreuses mesures.

\section{Les résultats.}

11. Nickel. - Nous avons mesuré les valeurs des termes $V_{u .3 \%}$ pour les plans 111, 200, 220, 311, 331 et 420 .

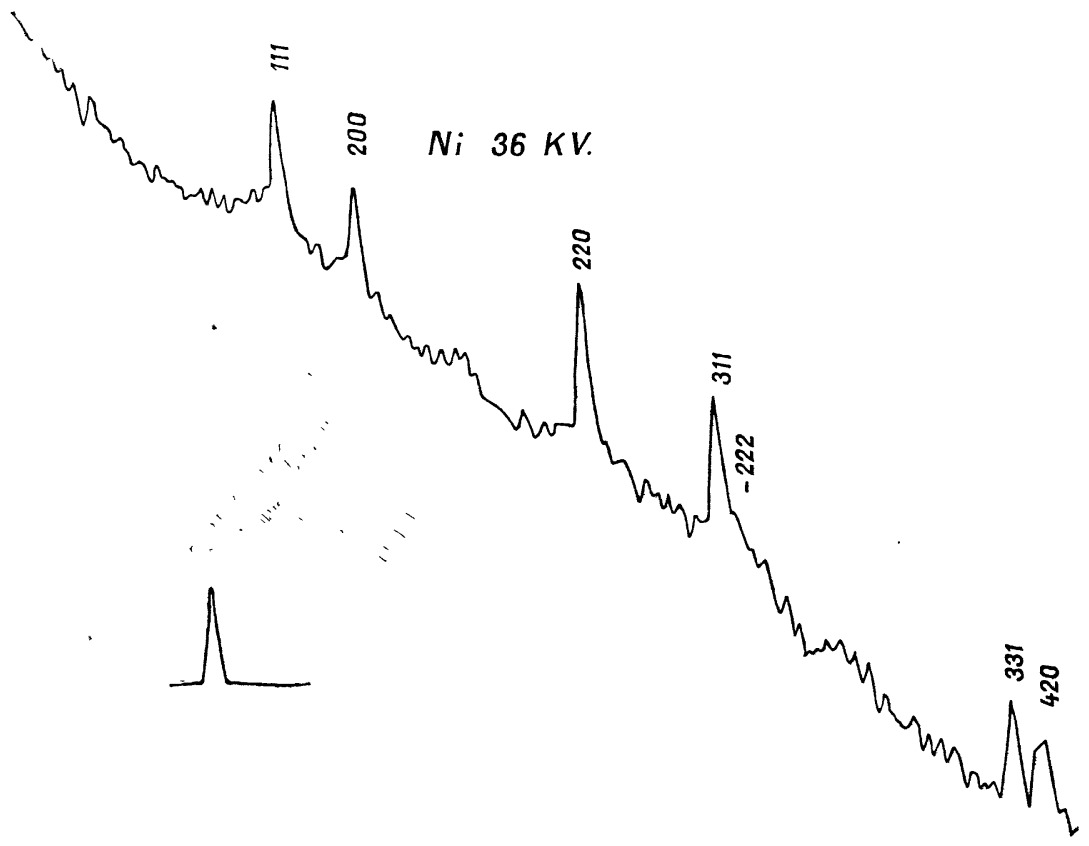

Fig. 4.

$E=36 \mathrm{kV}$.

\begin{tabular}{|c|c|c|c|c|c|c|}
\hline PLANS & .111 & 200 & 220 & 311 & 331 & 420 \\
\hline$\Delta r \ldots$ & $0,009 \mathrm{~cm}$ & 0,008 & 0,010 & 0,010 & 0,010 & 0,010 \\
\hline$r \ldots$. & $0,955 \mathrm{~cm}$ & 1,10 코․ & 1,56 & 1,827 & 2,23 & 2,32 \\
\hline
\end{tabular}

Erreur maximum possible sur $r: 0,008 \mathrm{~cm}$.

Or. $E=41,6 \mathrm{kV}$.

\begin{tabular}{|c|c|c|c|c|}
\hline pLANs & 111 & 200 & 220 & 311 \\
\hline$\Delta r \ldots$ & $0,014 \mathrm{~cm}$ & 0,010 & 0,008 & 0,008 \\
\hline$r \ldots \ldots$ & $0,775 \mathrm{~cm}$ & 0,892 & 1,265 & 1,477 \\
\hline
\end{tabular}

Erreur possible sur $\lrcorner r: 0,007 \mathrm{~cm}$.

\begin{tabular}{|c|c|c|c|c|}
\hline PLANS & 111 & 200 & 220 & 311 \\
\hline$\Delta r \ldots \ldots$ & $0,026 \mathrm{~cm}$ & 0,022 & 0,022 & 0,022 \\
\hline$r \ldots$ & $0,725 \mathrm{~cm}$ & 0,84 & 1,185 & 1,389 \\
\hline
\end{tabular}

Graphite. $\quad E=34,6 \mathrm{kV}$.

\begin{tabular}{|c|c|c|c|}
\hline PLAks & $100+101$ & 110 & $200+202$ \\
\hline$\Delta r . \ldots \ldots$ & $0,1 \mathrm{~cm}\left(^{*}\right)$ & 0001 & $(0,04)$ \\
\hline$r \ldots \ldots \ldots$ & $0,9 \unlhd \mathrm{cm}$ & 1,598 & 1,9 \\
\hline
\end{tabular}

(*) Comme l'ont montré Finch et Wilman (Proc. Roy. Soc., London, A. 1936 , vol. 155, p. 66) cet anneau est complexe; il résulte en effet des réflexions sur les plans 100 et 111 e 1 comporte en plus des " extra-rings ". ll en est de même de l'anneau 200, qui est d'ailleurs le second ordre du précédent. 
Intéressons-nous spécialement aux mesures portant sur les métaux. L'application de la formule (14) donne les valeurs des termes $V_{a, 3 \gamma}$. Les erreurs de mesure sur $\Delta \theta$ sont nécessairement des erreurs par excès car toutes les causes d'erreurs contribuent à élargir les anneaux (et surtout la variation de tension); nous prendrons donc pour valeur de $\Delta \theta$ le nombre résultant de la différence entre $\Delta r$ et l'erreur.

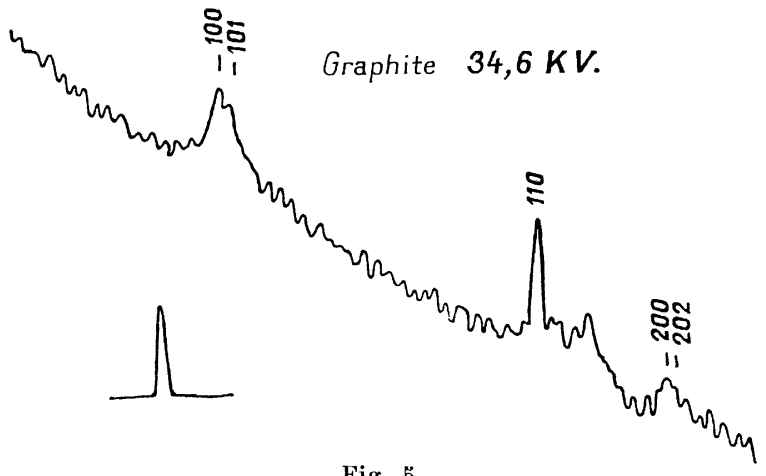

Fig. 5 .

L'application de la formule (9) donne alors un ordre de grandeur acceptable pour les plans d'indice supérieur à 220 ; par exemple, pour $\mathrm{Ni}$, on trouve :

$$
\begin{aligned}
& V_{341}=39 \mathrm{~V} \\
& V_{331}=32 \mathrm{~V} \\
& V_{420}=30 \mathrm{~V} .
\end{aligned}
$$

Ces nombres sont cependant trop élevés (voir $\S 3$ ). Pour Au, on a :

$$
V_{220}=33 \mathrm{~V} ; \quad V_{311}=28 \mathrm{~V} \text {. }
$$

Pour le graphite, on trouve :

$$
V_{110}=21 \mathrm{~V} \text {. }
$$

Il suit de là que la méthode expérimentale utilisée, tout en donnant un ordre de grandeur de la valeur des termes $V_{a, \beta y}$, conduit cependant à des valeurs qui peuvent être deux à trois fois plus élevées que celles qui résultent des calculs théoriques indiqués au § 3 .

12. Conclusions. - Les principaux résultats de celte étude sont les suivants :
1 La mesure des largeurs des anneaux de diffraction électronique des cristaux ne s'accorde pas avec la théorie actuelle de la réflexion des ondes électroniques par les cristaux; celle-ci assignerait, en effet, d'après Bethe et Kikuchi, aux termes $V_{\alpha, j}$ du développement en série de Fourier du potentiel interne du cristal une valeur donnée par (13); la valeurdes $V_{\alpha \beta \beta}$ ainsi calculée serait de l'ordre d'une petite fraction de volt alors que les valeurs des termes $V_{u, 3 y}$ sont pour les métaux au moins de l'ordre d'une dizaine de volts (pour les valeurs faibles des indices $\alpha, \beta, \gamma$ ).

Les auteurs proposent, au lieu de la formule (13), la formule (14) directement déduite de la théorie des métaux.

$2^{\circ}$ Une étude aussi poussée que possible de la technique expérimentale a permis de mesurer les valeurs de $\Delta \theta$ et, par conséquent, les valeurs de $V_{a \beta \beta \%}$. On obtient ainsi l'ordre de grandeur de ces termes; les erreurs expérimentales restent cependant suffisantes pour justifier d'un écart assez considérable.

$3^{\circ}$ De cette étude se dégagent eependant les conditions expérimentales qu'il conviendrait de réaliser pour obtenir les valeurs correctes des termes $V_{\alpha \beta \gamma}$. Il y aurait lieu en effet de reprendre des mesures analogues à celles que nous avons faites avec des électrons moins rapides. On vérifie aisément, en effet, d'après la formule (14) que la largeur $\Delta \theta$ des anneaux de diffraction augmente comme $\frac{1}{3}$; en opérant avec des électrons $\boldsymbol{E}^{\overrightarrow{2}}$

de quelques kilovolts, on pourrait donc espérer obtenir des mesures très satisfaisantes de $\Delta \theta$, donc de $V_{\alpha, 3 \gamma}$. On pourrait en mème temps vérifier la validité de la formule (14) en étudiant systématiquement la variation de $\Delta \theta$ en fonction de $E$. Il faudrait cependant opérer avec des électrons ayant des énergies de quelques $\mathrm{kV}$ au moins; car pour des électrons lents, l'absorption peut intervenir pour donner un élargissement supplémentaire des anneaux de diffraction (ainsi que l'a montré J. Farineau, article en publication dans ce mème numéro du Journal de Physique, p. 15̃T).

D'autre part, il y aurait lieu d'opérer sous une tension rigoureusement constante; en effet, dans nos mesures, la variation de tension bien qu'extrêmement petite, paraît être la principale source d'erreur. Des essais dans ce sens sont actuellement en voie de réalisation.

Manuscrit reçu le 31 janvier 1938.

\section{BIBLIOGRAPHIE}

(1) Leip-iger l'ortrüge, 1930, p. 5ั.

(`) Leipziger Vorträge, 1939 , p. 5

(3) Cf. p. ex. L. Brillours, Stat. Quant., t. II, p. 296.

(4) Bethe, Ann. der P'hys., 1928, 87, 55.
(5) Kıкuchı. Scient. Pap. Inst. Phys. Chem. Res. Tokyo; 1933, 26,225 .

(6) Watson. Phil. Mag., 1927, 3, 849. 